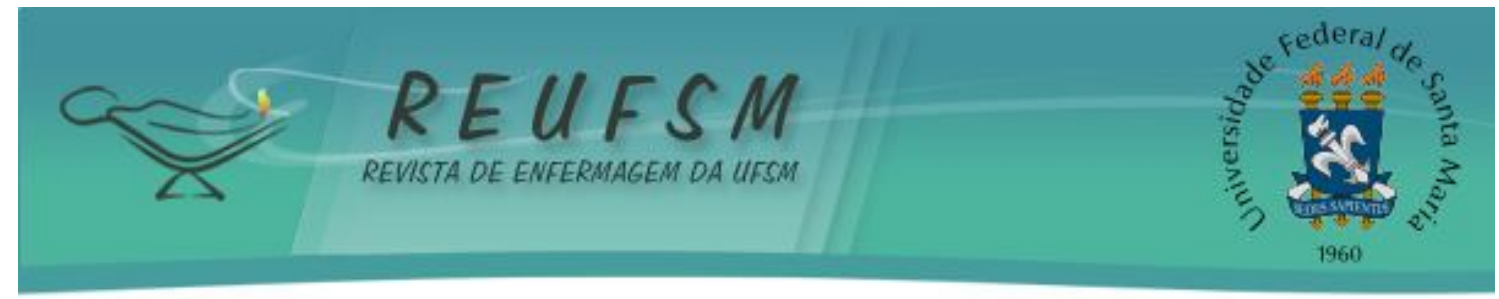

ARTIGO ORIGINAL

\title{
OFERTA DE SERVIÇOS ASSISTENCIAIS AOS ADOLESCENTES EM SEIS MUNICÍPIOS DA 12a COORDENADORIA REGIONAL DA SAÚDE ${ }^{1}$
}

\author{
HEALT H CARE SERVICES TO ADOLESCENTS IN SIX CITIES OF THE 12nd REGIONAL \\ HEALT H COORDINATION
}

\section{OFERTA DE SERVICIOS DE ASSISTENCIA A LOS ADOLE SCENTES EN SEIS MUNICIPIOS DE LA 12a COORDINADORÍA REGIONAL DE SALUD}

\author{
Luciana Griebeler Oliveira ${ }^{2}$ \\ Zaléia Prado de Brum ${ }^{3}$ \\ Stefanie Griebeler Oliveira ${ }^{4}$ \\ Simone Wünsch ${ }^{5}$ \\ Raquel Pötter Garcia ${ }^{6}$ \\ Bruna Sodré Simon ${ }^{7}$ \\ Dyan Jamilles Teixeira Brum ${ }^{8}$
}

RESUMO: Este estudo objetivou conhecer a oferta de serviços de atenção aos adolescentes nos municípios da $12^{\mathrm{a}}$ Coordenadoria Regional de Saúde (CRS), na percepção dos enfermeiros e conselheiros tutelares. Tratou-se de uma pesquisa qualitativa. A amostra foi constituída de conselheiros tutelares e enfermeiros de seis dos 25 municípios de abrangência da 12a ${ }^{\text {CRS }}$ do Rio Grande do Sul. Os dados foram coletados por meio de entrevista semiestruturada. Os dados foram organizados e posteriormente realizou-se a análise de conteúdo. Após essa etapa, emergiram duas categorias: Oferta de Serviços aos Adolescentes; e Oferta de Serviços para Adolescentes em Situação de Vulnerabilidade Social. Muitas ações são direcionadas aos adolescentes no âmbito da saúde, e nos âmbitos educativo, religioso ou social, todavia, na

\footnotetext{
${ }^{1}$ Artigo elaborado na disciplina de Trabalho de Conclusão de Curso de Enfermagem da Universidade Regional Integrada do Alto Uruguai e das Missões (Campus de Santo Ângelo).

${ }^{2}$ Enfermeira. Especialista em Saúde Coletiva com Ênfase em Saúde da Mulher. Estratégia Saúde da Família Doutor Chico/Secretaria Municipal de Saúde de São Luiz Gonzaga/RS. Membro do Grupo de Pesquisa Cuidado, Saúde e Enfermagem da Universidade Federal de Santa Maria (UFSM). lucianagoenf@hotmail.com

${ }^{3}$ Enfermeira. Mestre em Educação. Professora do Curso de Enfermagem da Universidade Regional Integrada do Alto Uruguai e das Missões (Campus de Santo Ângelo). Membro do Grupo de Estudos e Pesquisa em Educação Saúde Enfermagem (GEPESE). zaleia@santoangelo.uri.br

${ }^{4}$ Enfermeira. Mestre em Enfermagem. Doutoranda em Enfermagem pela Universidade Federal do Rio Grande do Sul (UFRGS). Bolsista CAPES. Membro dos Grupos de Pesquisa: Cuidado Saúde e Enfermagem (UFSM); e, Grupo de Estudos Culturais na Educação em Saúde e Enfermagem (UFRGS). stefaniegriebeler@yahoo.com.br

${ }^{5}$ Enfermeira. Especialista em Saúde Pública. Mestranda em Enfermagem (UFSM). Setor de Vigilância Sanitária/Secretaria Municipal de Saúde de São Luiz Gonzaga/RS. Membro do Grupo de Pesquisa Cuidado, Saúde e Enfermagem (UFSM). simone.wunsch@gmail.com

6 Enfermeira. Mestranda em Enfermagem (UFSM). Membro do Grupo de Pesquisa Cuidado, Saúde e Enfermagem (UFSM). raquelpotter_@hotmail.com

7 Enfermeira. Membro do Grupo de Pesquisa Cuidado Saúde e Enfermagem (UFSM). bru.simon@hotmail.com

${ }^{8}$ Acadêmica de Enfermagem (UFSM). Bolsista do Programa de Educação Tutorial (PETEnfermagem). Membro do Grupo de Pesquisa Cuidado, Saúde e Enfermagem (UFSM). jamillesenf@gmail.com
} 


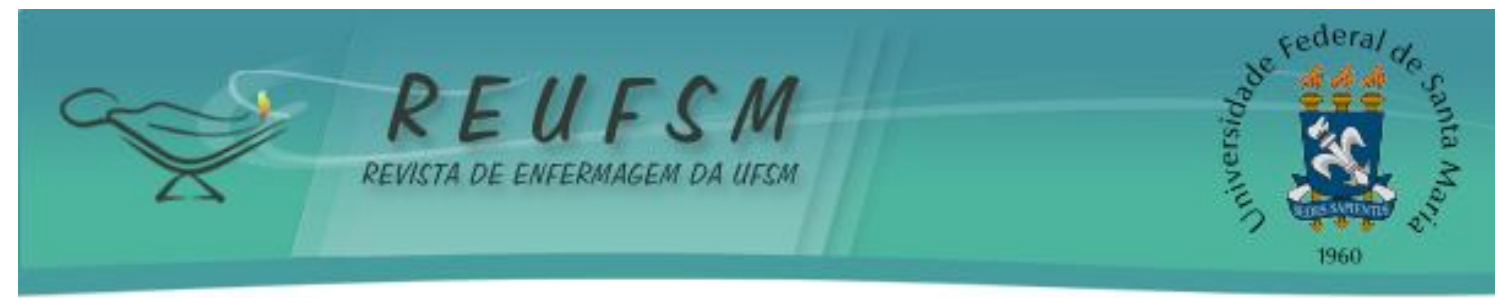

maioria dos municípios, essas ações não são articuladas. Foram apontadas também ações específicas para os adolescentes em vulnerabilidade social.

Descritores: Enfermagem; Adolescente; Serviços de saúde para adolescentes; Saúde do adolescente.

ABSTRACT: This study aimed to know the offer of services of health care to adolescents in the cities of the 12nd Regional Health Coordlnation (RHC), through the perception of nurses and tutelary counselors. It is a qualitaive research. The sample was constituted by tutelary counselors and nurses of six, out of 25 cities of the 12nd RHC of Rio Grande do Sul. Data was collected through semi-structured interviews and then organized to proceed to content analysis, which was later carried out. After such stage, two categories emerged: Offer of Services to Adolescents and Offer of Services to Adolescents in Social Vulnerability. Several actions are directed to adolescents, as concerns the health, educational, religious or social fields. However, in most of the cities such actions are not articulated. Specific actions towards adolescents in social vulnerability were highlighted.

Descriptors: Nursing; Adolescent; Adolescent health services; Adolescent health.

RESUMEN: Este estudio objetivó conocer la of erta de servicios de atención a los adolescentes en los municipios de la $12^{\text {a }}$ Coordinadoría Regional de Salud (CRS), en la percepción de los enfermeros y consej eros tutelares. Es una pesquisa cualitativa. La muestra fue constituída de consej eros tutelares y enfermeros de seis de los 25 municipios de cobertura de la $12{ }^{\mathrm{a}} \mathrm{CRS}$ del Rio Grande del Sur. Los datos fueron colectados a través de entrevista semiestructurada. Registrados y posteriormente se realizó el análisis del contenido. Después de esa etapa, emergieron dos categorías: Oferta de Servicios a los Adolescentes; y Oferta de Servicios para Adolescentes en Situación de Vulnerabilidad Social. Muchas acciones son direccionadas a los adolescentes en los ámbitos de salud, de educación, de religión o social, en la mayoría de los municipios, esas acciones no son articuladas. Fueron apuntadas también acciones específicas para adolescentes en vulnerabilidad social.

Descriptores: Enfermería; Adolescente; Servicios de salud para adolescentes; Salud del adolescente.

\section{INTRODUÇÃO}

A adolescência é entendida como um período no qual o indivíduo passa por transformações biopsicossocioculturais e que constitui um processo fisiológico da maturação humana. ${ }^{1}$ Nessa fase podem surgir muitas questões inquietantes para 0 adolescente, pois este começa a ter enfrentamentos em relação aos limites impostos pelos seus pais, o que pode gerar conflitos. Ele também descobre-se sexualmente por meio do namoro, das transformações sexuais e dos riscos com doenças sexualmente transmissíveis (DSTs). Com a pouca experiência, pode envolver-se com situações inesperadas, como gravidez não planejada, doença sexualmente adquirida, e uso de drogas. ${ }^{2-3} \mathrm{~A}$ fase da adolescência requer uma atenção especial, pois o adolescente necessita da orientação de adultos, que pode ser desenvolvida pelo enfermeiro por meio de ações de saúde. ${ }^{4}$

O Ministério da Saúde propõe diretrizes para organização dos serviços de atenção aos adolescentes. Tais proposições são facilmente adaptáveis e flexíveis aos diversos locais do extenso território brasileiro. Ainda, destaca-se a necessidade da existência de serviços de saúde de qualidade, o que implica em um desafio para o alcance de melhores condições de vida e de saúde dos adolescentes e jovens brasileiros. ${ }^{5}$

Alguns estudos ${ }^{6-8}$ discutem entre profissionais de saúde a oferta dos serviços de saúde para 0 adolescente. Em um desses estudos ${ }^{8}$, foi identificado que a maior parte dos adolescentes que buscam os serviços de saúde não possuem queixas clínicas específicas. Todavia, o modelo de atenção que esse público encontra é o que privilegia os 


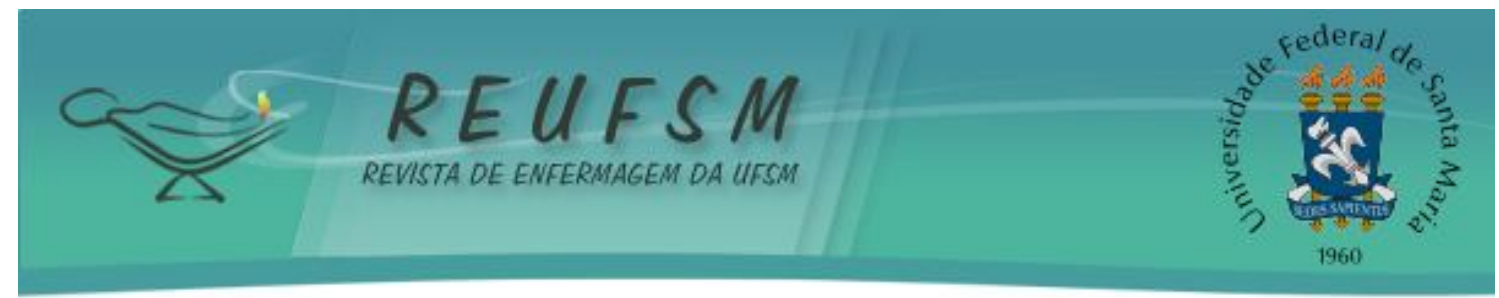

procedimentos clássicos, como anamnese e exame físico. Essa forma de atenção aos adolescentes pode fazer com que suas demandas não sejam atendidas, afastando-os, assim, dos profissionais de saúde. ${ }^{8}$ As demandas incluem orientações que abordem os temas inquietantes e pertinentes para a experiência do adolescer, tais como a sexualidade, drogas, autoestima, relações interpessoais, entre outros.

Além disso, outros dois estudos apontam as dificuldades que os profissionais de saúde possuem em se organizar para trabalhar com essa população específica e suas demandas. Salientarse também a falta de preparo para atuar junto a um público crítico e questionador, com muitas inquietudes. ${ }^{6-7}$

Diversos relatos de experiência com grupos de adolescentes, em especial, realizados por enfermeiros, já foram publicados. Grande parte aborda as questões de sexualidade e DSTS/ IIDS $^{2,9-10}$, e ressalta as potencialidades das metodologias participativas com esse público, pois as mesmas permitem uma construção do conhecimento a partir da troca entre os integrantes do grupo, incluindo o profissional que facilita as atividades.

Percebe-se que, apesar das pesquisas ${ }^{6-8}$ demonstrarem dificuldades de estrutura tanto física quanto de recursos humanos para atender esses grupos, existem trabalhos ${ }^{2,9-10}$, mesmo que de forma isolada, que buscam atender as necessidades dessa fase de vida. Tendo em vista a importância de uma atenção especializada, questionou-se como é a oferta de serviços de atenção para essa faixa etária. Desse modo, este trabalho teve como objetivo conhecer a oferta de serviços de atenção aos adolescentes nos municípios da $12^{\mathrm{a}}$ Coordenadoria Regional de Saúde (CRS) do Rio Grande do Sul (RS), na percepção dos enfermeiros e conselheiros tutelares.

\section{MÉTODO}

Trata-se de uma pesquisa qualitativa. A escolha pela pesquisa qualitativa deu-se pela busca do conhecimento dos serviços de atenção ofertados aos adolescentes, na percepção dos enfermeiros e conselheiros tutelares. A amostra foi constituída de consel heiros tutelares e enfermeiros dos 25 municípios de abrangência da $12^{a}$ CRS do RS. A opção pelos enfermeiros justifica-se por ser característica dessa região o encargo de coordenação das unidades de saúde da família. Desse modo, no âmbito da saúde, esses profissionais possuem o conhecimento das ações desenvolvidas pelo serviço de saúde. A escolha dos conselheiros tutelares remete-se ao âmbito social, e de sua atuação junto aos problemas sociais que envolvem os adolescentes. A amostra constituiu-se de um enfermeiro que atuasse em Unidade Básica de Saúde, indicado pelo Secretário Municipal de Saúde, e um membro do Conselho Tutelar, indicado pelo presidente do mesmo, de cada município. Ambos, enfermeiro e conselheiro, deveriam ter mais de um ano de atuação nos espaços especificados. Os que não se incluíam nesses critérios foram excluídos da pesquisa.

0 período de coleta de dados ocorreu de março a maio de 2008. Realizou-se contato prévio por e-mail com os profissionais de cada cidade, já informando-os sobre a pesquisa, os objetivos da mesma, e, ainda, com a remessa da entrevista semiestruturada, mas não houve retorno dos mesmos. Essa dificuldade foi contornada quando a pesquisadora decidiu dirigir-se a alguns municípios. 0 critério para escolha destes foi pela proximidade geográfica. Ao todo, seis municípios responderam a pesquisa, somando seis enfermeiros e seis conselheiros tutelares.

Os dados foram coletados por meio de entrevista semiestruturada, em encontro previamente agendado. Essa entrevista tinha como pontos principais: descrição dos serviços de atenção of ertados aos adolescentes; percepção dos entrevistados em relação a esses serviços ofertados; limitações, conquistas, potencialidades desses serviços; percepção dos entrevistados em relação à aceitação dos adolescentes a esses serviços.

A entrevista foi conduzida por meio de uma estrutura solta, que consiste em questões abertas que definem a área a ser explorada e a partir das quais o entrevistador ou o entrevistado podem divergir, a fim de prosseguir com uma ideia ou uma resposta com 


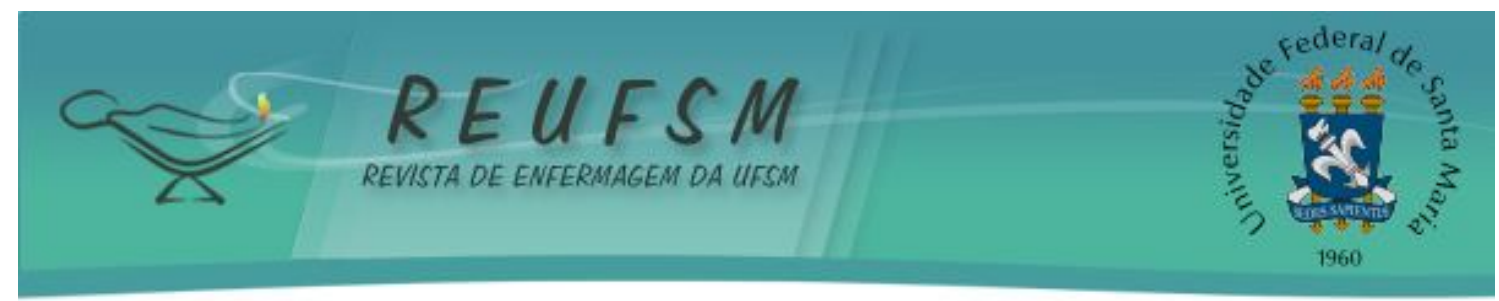

maiores detalhes. ${ }^{11} \mathrm{O}$ registro dos dados foi realizado pelo pesquisador, no documento que continha as questões.

De posse de todos os dados, estes foram organizados, para a realização da análise. Utilizou-se a análise de conteúdo ${ }^{12}$, que se define pela explicitação do sentido contido no documento e/ ou o modo em que este pode ser transformado com a finalidade de oferecer um significado, levando-se em conta a frequência da repetição dos termos, o aparato e andamento do discurso. ${ }^{12}$ Os dados foram organizados por aproximação de ideias em um parágrafo, que explicitava como os suj eitos percebiam a oferta de serviços de atenção aos adolescentes.

Respeitando os aspectos éticos, conforme a Resolução no 196/96. ${ }^{13}$, que regulamenta a pesquisa envolvendo seres humanos, foi disponibilizado o Termo de Consentimento Livre e Esclarecido aos sujeitos de pesquisa, e a coleta de dados ocorreu com a aprovação do Comitê de Ética em Pesquisa da Universidade Regional Integrada Campus Santo Ângelo, sob o número 018-04/ TCH/ 08.

\section{RESULTADOS E DISCUSSÃO}

Após análise de conteúdo dos dados obtidos com a pesquisa, emergiram duas categorias: Oferta de Serviços aos Adolescentes, e Adolescentes em Vulnerabilidade Social.

\section{Oferta de serviços aos adolescentes}

A oferta de atividades que visem melhorar a qualidade de vida dos adolescentes se fazia presente nos municípios pesquisados, conforme os depoimentos da grande maioria dos enfermeiros e conselheiros tutelares. Desse modo, pode-se pensar que havia oferta, mas alguns profissionais desconheciam esses serviços, pois relataram que havia ausência dessas atividades em alguns municípios. Também destaca-se que, no mesmo município, um dos profissionais dizia que havia serviços de atenção ao adolescente, e o outro apresentava desconhecimento das ações direcionadas a esse público. Acredita-se, desse modo, que existisse pouca articulação entre os espaços de saúde e os espaços sociais, por haver essa contradição nas informações.

Entre as atividades of ertadas, tanto nos espaços de saúde quanto nos espaços de atenção no âmbito social, para atender as necessidades dos adolescentes, promovendo qualidade de vida, na percepção dos enfermeiros e dos conselheiros tutelares, estavam: educação em saúde nas escolas; escolas de informática; atividades esportivas; grupos de adolescentes realizados por enfermeiros; teatro; atividades promovidas pelo Centro de Tradições Gaúchas (CTG); grupos de jovens nas igrejas; capoeira.

Percebeu-se que tanto os enfermeiros quanto os conselheiros tutelares citaram atividades não necessariamente ligadas aos seus campos de atuação. Por outro lado, observa-se que os mesmos não consideraram atendimentos individuais aos adolescentes, que, certamente, ocorriam no cotidiano de seu trabalho. Já, em outro estudo ${ }^{6}$, os profissionais de saúde demonstraram o reconhecimento das atividades realizadas no âmbito individual nas unidades de saúde, como consultas de enfermagem, consultas médicas e orientações na entrega de métodos contraceptivos.

Apenas um dos municípios possuía um projeto de ações de saúde ao adolescente que ocorria de forma sistematizada, com encontros programados, tanto na escola, quanto na unidade de saúde. Assim, a falta de sistematização das ações e de planejamento para o atendimento das demandas também podem ser consideradas como fatores que prejudicam o acesso dessa população aos serviços de atenção a saúde.

Diante desses dados que ilustram a of erta dos serviços de atenção aos adolescentes, pode-se pensar uma aproximação com o componente programático da vulnerabilidade, com os serviços não sendo disponibilizados para todos, ocorrendo certa vulnerabilidade na sua 


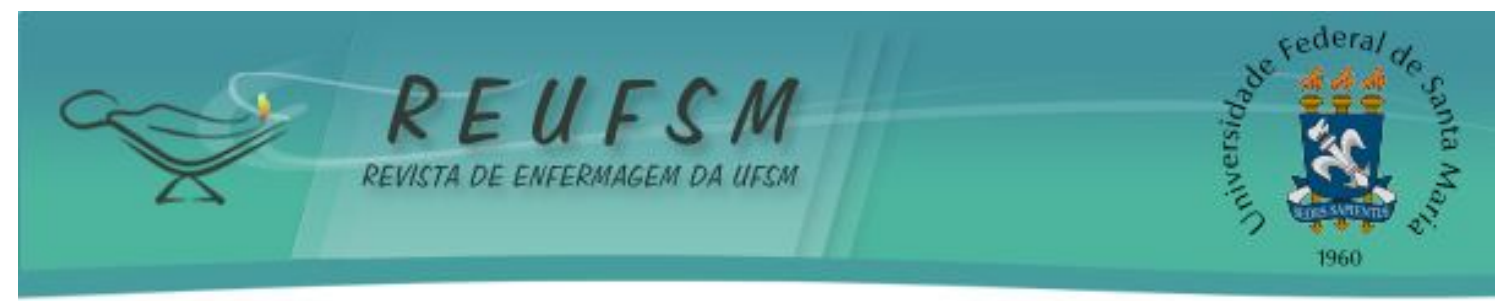

organização e consequentemente para os adolescentes que não têm acesso. 0 componente programático envolve o grau e a qualidade de compromisso, recursos, gestão e monitoramento de programas de prevenção e cuidado, os quais são relevantes para identificar necessidades, canalizar os recursos sociais existentes e otimizar seu uso. Ainda, permite situar os aspectos educativos para além do caráter normativo e centrado no objeto, levando-se a articular as intervenções em saúde e as ações programáticas. ${ }^{14}$ Desse modo, na realidade estudada pode-se afirmar que havia vulnerabilidade nessas questões, pois as ações ainda apresentavam-se desarticuladas e isoladas, dificultando a oferta de serviços aos adolescentes, o que interferiria na sua qualidade de vida.

Ainda, grande parte dos enfermeiros desta pesquisa manifestou limitações para o desenvolvimento das atividades para os adolescentes, entre elas: 0 desinteresse do adolescente em participar de ações de saúde; a falta de planejamento das ações e de implantação das atividades previstas pelo Ministério da Saúde destinadas aos adolescentes. $\mathrm{Na}$ visão destes enfermeiros entrevistados, o adolescente acredita que tem capacidade para resolução dos seus problemas e não se permite escutar seus pais, que muitas vezes possuem preocupações com seus filhos. Essa situação, conforme os entrevistados, termina por gerar conflitos entre pais e filhos. Ressalta-se que esta percepção em relação aos adolescentes, de se sentirem "autossuficientes" em suas experiências, precisa ser repensada, pois os adolescentes se sentem mais bem amparados quando possuem a referência de um adulto para escutá-Ios e proporcionar orientações. Todavia, vai depender do tipo de abordagem que este adulto irá fazer, para que o adolescente o vej a como mentor.

Em contrapartida a essa percepção dos enfermeiros, os conselheiros tutelares expuseram que os adolescentes possuíam interesse pelas atividades disponibilizadas. Além disso, ressaltaram que, quando há envolvimento do adolescente nas atividades ofertadas, ocorre redução de violência, prevenção de envolvimento com drogas, bom desempenho escolar, bom relacionamento familiar e convívio com os amigos. Outro estudo ${ }^{15}$ salientou que a participação em grupos, além de permitir uma integração e produção de redes sociais, também pode amenizar problemas com dependência química.

Diante disso, pode-se pensar esses dados proximando-os aos componentes individual e social da vulnerabilidade. 0 individual consiste fundamentalmente na ordem cognitiva e na ordem comportamental. A ordem cognitiva refere-se à quantidade e qualidade de informação de que os indivíduos dispõem e a capacidade que possuem de elaborá-la. Já a ordem comportamental, remete-se à capacidade, habilidade e interesse para transformar essas preocupações em atitudes e ações protegidas e protetoras. ${ }^{14}$

0 componente social da vulnerabilidade envolve 0 acesso às informações, as possibilidades de processá-las e o poder de incorporá-las a mudanças práticas na vida cotidiana, condições estas inteiramente associadas ao acesso a recursos materiais, a instituições sociais, à possibilidade de enfrentar barreiras culturais e de estar livre de coerções violentas de todas as ordens. ${ }^{14} \mathrm{Na}$ realidade estudada, havia certa presença da vulnerabilidade, considerando esses componentes também, pois os profissionais apontaram dificuldades e limitações na organização e execução de ações com esse público específico, que, por sua vez, também termina por ficar em situação de vulnerabilidade. Isto é, se não há serviços que deem suporte aos adolescentes, eles podem ficar desassistidos, ficando assim à mercê dos acontecimentos não desejados para essa fase, como adoecimentos que vão desde adquirir doenças sexualmente transmissíveis ao envolvimento de drogas. Todavia, apesar de alguns relatos trazerem serviços de apoio aos adolescentes, mesmo assim a fragilidade presente parece ser maior, pois não foi possível detectar uma articulação entre esses serviços, o que restringe os adolescentes na melhoria de suas condições, tornando-os vulneráveis. 


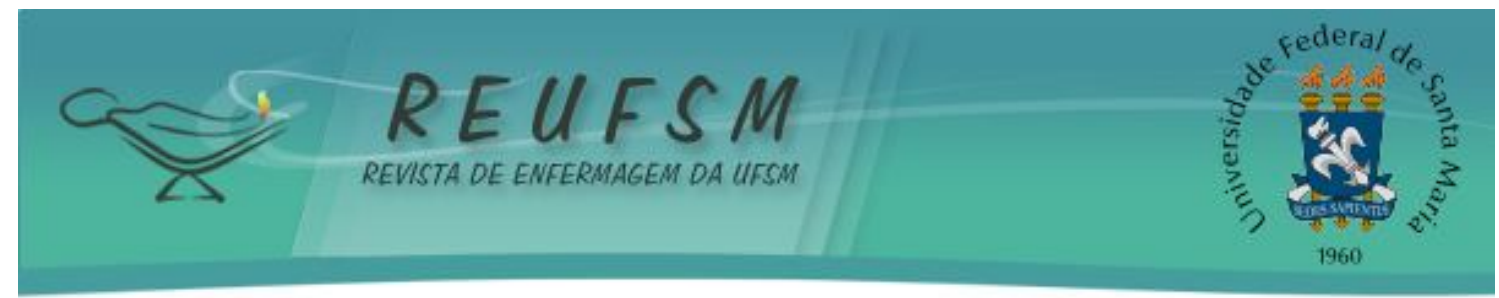

Oferta de Serviços para Adolescentes em Situação de Vulnerabilidade Social

Em relação aos adolescentes em situação de vulnerabilidade social (usuários de drogas, envolvidos com violência), esses indivíduos são tratados pela sociedade algumas vezes com indiferença, e também são suscetíveis a preconceitos.

Segundo os entrevistados, os municípios procuravam cumprir com suas obrigações sociais, para que esses adolescentes não fossem discriminados. Em caso de violência ou risco, eles eram encaminhados para a Delegacia de Polícia; na ocorrência de muitos conflitos familiares propiciava-se assistência à família e, no caso de os adolescentes não serem aceitos mais pela família, eles eram encaminhados para abrigos. Além disso, faziase necessário conscientizar as pessoas de que o jovem necessitava de ajuda psíquica, social, cultural, afetiva, e que o preconceito tornava-se prejudicial diante da situação. Nesse sentido, os municípios ofereciam auxílio, como tratamentos em clínicas especializadas, em caso de envolvimento com drogas, como também para a família, por meio de oficinas terapêuticas no Centro de Atenção Psicossocial - Álcool e Drogas. A partir disso, percebe-se que havia certa estrutura para atendimento dos adolescentes em vulnerabilidade, mas talvez, de algum modo, com pouca articulação.

Diante desses dados, pode-se pensar na articulação dos três componentes da vulnerabilidade: individual, social e programático. A articulação desses três el ementos permite um entendimento, aos profissionais dos espaços de saúde e dos espaços de ação social, de que as pessoas não são vulneráveis, mas podem estar ou estão em situação de vulnerabilidade. ${ }^{14}$

Um estudo revela que os enfermeiros, como promotores da saúde, necessitam elaborar novas alternativas preventivas e maneiras diferenciadas de abordar a temática das drogas, de modo mais próximo à realidade dos jovens. ${ }^{16}$ Dessa forma, estar atento a essa parcela da população torna-se relevante, uma vez que, diante da atual sociedade globalizada, os riscos de problemas advindos dessas substâncias tornam-se maiores.

A religião também possui papel fundamental nessa fase. Entre os incentivos utilizados pelos organizadores das atividades religiosas, para o desenvolvimento do interesse religioso pelo jovem, tem-se a realização de missas, cultos e atividades religiosas, grupo de jovens e de famílias em igrejas, escolas, trabalhos voltados para a promoção da espiritualidade. A religião pode intervir no desenvolvimento de seus princípios, valores, crenças, e é de relevância para uma vida salutar no adolescer.

Os adolescentes que relataram pertencer a uma religião apresentaram níveis mais altos de bem-estar psicológico. ${ }^{17}$ No entanto, os adolescentes que tinham menor envolvimento religioso apresentam-se psicologicamente afetados. ${ }^{18} 0$ fato de ter uma religião também interfere no envolvimento com as drogas, pois pesquisadores revelam que, quando os jovens estão inseridos e frequentam uma religião, menores são as chances de envolvimento com drogas.

\section{CONSIDERAÇÕES FINAIS}

Percebe-se que os municípios encontravam dificuldade na realização de trabalhos intersetoriais, uma vez que as respostas de enfermeiros e conselheiros tutelares apresentavam algumas divergências. Acredita-se que, para que haja um resultado efetivo, tem de haver o trabalho em equipe, o trabalho interdisciplinar, que envolva os campos da saúde, da educação e o social. Todavia, no contexto estudado, verifica-se que existiam algumas atividades/trabalhos voltados para adolescentes, porém estes se encontravam fragmentados, evidenciando a ausência da intersetorialidade.

Com as análises dos resultados, observou-se o quanto é prejudicial não haver o conhecimento das ações existentes nos municípios por todos os profissionais, no que diz respeito às ações de atenção à saúde ao adolescente, pois havia contradição nas respostas obtidas. Compreende-se que muitas 


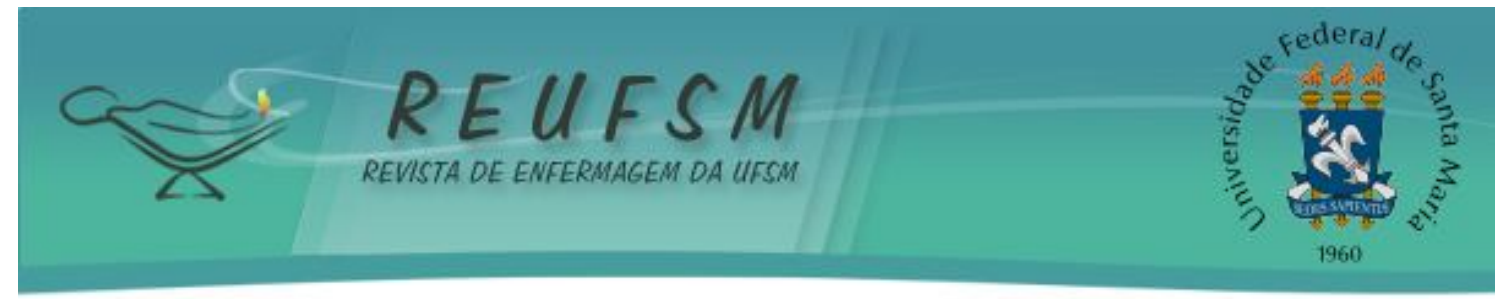

atitudes e planejamentos devam ser mudados, melhorados, reconhecidos por parte da ação social, pois é através deles que se pode beneficiar a população jovem.

0 estudo apresenta algumas limitações, mas permite um desenho da oferta dos serviços de atenção aos adolescentes nos municípios pesquisados. Tais limitações referemse ao fato de apenas seis municípios terem sido entrevistados, bem como apenas enfermeiros e conselheiros tutelares terem sido sujeitos da pesquisa. Desse modo, recomenda-se a realização de outros estudos acerca do tema, para que as ações voltadas aos adolescentes sejam visualizadas e estendidas para outros locais.

\section{REFERÊNCIAS}

1. Ressel LB, Sehnem GD, J unges CF, Hoffmann IC, Landerdahl MC. Representações culturais de saúde, doença e vulnerabilidade para mulheres adolescentes. Esc Anna Nery Rev Enferm. 2009; 13(3):552-7.

2. Oliveira SG, Ressel LB. Grupos de adolescentes na prática de enfermagem: um relato de experiência. Ciênc cuid saúde. 2010; 9(1):144-8.

3. Oliveira SG, Domingues IB, Wünsch $\mathrm{S}$. Consulta de enfermagem aos adolescentes em uma unidade básica de saúde. Nursing (São Paulo). 2010;140:35-8.

4. Ramos FRS, Pereira SM, Rocha CRM. Viver e adolescer com qualidade In: Ramos FRS (org). Adolescer: compreender, atuar e acolher. Brasília: ABEn: MS; 2001. p.12-31.

5. Brasil. Ministério da Saúde. Secretaria de Atenção à Saúde. Saúde integral de adolescentes e jovens: orientações para a organização de serviços de saúde. Brasília: Ministério da Saúde; 2005.

6. Ferrari RAP, Thomson Z, Melchior R. Atenção à saúde dos adolescentes: percepção dos médicos e enfermeiros das equipes da saúde da família. Cad saúde pública. 2006; 22(11):2491-95.

7. Formigli VLA, Costa COM, Porto L. Avaliação de um serviço de atenção integral à saúde do adolescente. Cad saúde pública. 2000; 16(3):831-41.

8. Ruzany MH, Swarcwald CL. Oportunidades perdidas de atenção integral ao adolescente: resultados do estudo-piloto. Adolesc Latinoam. 2000; 2(1):26-35.

9. Souza MM, Brunini S, Almeida NAM, Munari DB. Programa educativo sobre sexualidade e DST: relato de experiência com grupo de adolescentes. Rev bras enferm. 2007; 60(1): 102-5.

10. Hoga LAK, Abe CT. Relato de experiência sobre o processo educativo para a promoção da saúde de adolescentes. Rev Esc Enf USP. 2000; 34(4):407-12.

11. Pope C, Mays N. Pesquisa qualitativa na atenção à saúde. Artmed: Porto Alegre; 2006.

12. Turato ER. Tratado da metodologia da pesquisa clínico-qualitativa: construção teóricoepistemológica, discussão comparada e aplicação nas áreas da saúde e humanas. Petrópolis: Vozes; 2008.

13. Ministério da Saúde (BR). Conselho Nacional de Saúde. Diretrizes e normas regulamentadoras de pesquisa envolvendo seres humanos. Resolução $n$. 196, de 10 de outubro de 1996. Brasília; 1996.

14. Ayres JRCM, França-Júnior I, Calazans GJ, Saletti-Filho HC. O conceito de vulnerabilidade e as práticas de saúde: novas perspectivas e desafios. In: Czeresnia D, 


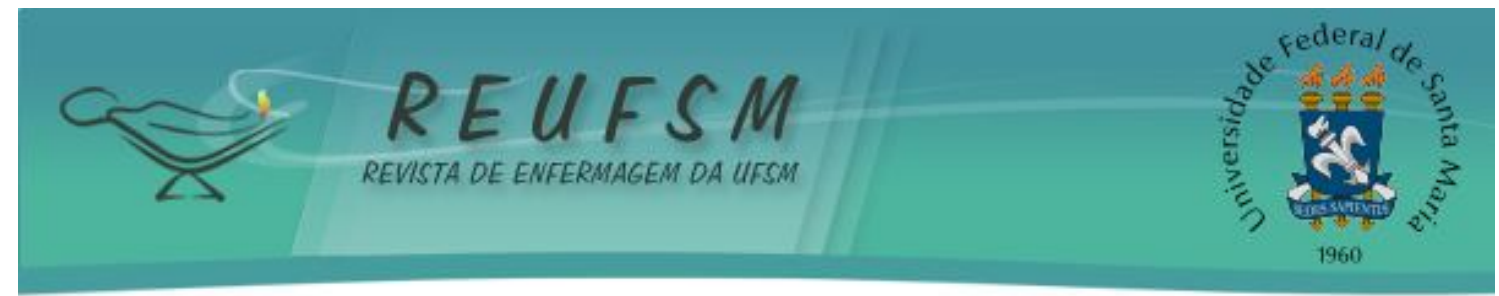

Freitas CM, organizadores. Promoção da saúde: conceitos, reflexões, tendências. Rio de J aneiro: Fiocruz; 2003. p. 117-39.

15. Budó MLD, Oliveira SG, Garcia RP, Simon BS, Schimith MD, Mattioni FC. Redes sociais e participação em uma comunidade referida a uma unidade de saúde da família. Rev gaúch enferm. 2010; 31(4): 753-60.

16. Cavalcante MBPT, Alves MDS, Barroso MGT. Adolescência, álcool e drogas: uma revisão na perspectiva da promoção da saúde. Esc Anna Nery Rev Enferm. 2008; 12(3):555-9.

17. Silva RA, Horta BL, Pontes LM, Faria AD, Souza LDM, Cruzeiro ALS et al. Bem-estar psicológico e adolescência: fatores associados. Cad saúde pública. 2007; 23(5):1113-8.

18. Dalgalarrondo P, Soldera MA, Filho Corrêa HR, Silva CAM. Religião e uso de drogas por adolescentes. Rev bras psiquiatr. 2004;26(2):82-90.

Data de recebimento: 20/08/2011

Data de aceite: 14/10/2011

Contato com autor responsável: Luciana Griebeler Oliveira

Endereço postal: Rua Álvaro Batista-1583, Bairro da Gruta. São Luiz Gonzaga/RS. CEP: $97800-000$

E-mail: lucianagoenf@hotmail.com 